\title{
Red-headed ash borer Neoclytus acuminatus acuminatus (Fabricius) (Coleoptera: Cerambycidae): the global distribution, current spreading and the seasonal activity depending on its different habitats
}

\author{
Sándor Keszthelyi ${ }^{1}$
}

Received: 14 January 2021 / Accepted: 9 March 2021 / Published online: 30 March 2021

(c) The Author(s) 2021

\begin{abstract}
Holarctic expansion of Neoclytus acumiantus acuminatus due to its polyphagous character has been a major concern for plant health, which raises several questions about its spreading directions. I have examined all bibliographical references, phytosanitary reports and authentic photographs from the online sources in the quest of the determination of the worldwide distribution and flight phenology of $N$. a. acuminatus. The expansion, including the western Palearctic regions as well as several Neotropical habitats, is proven and has become more intense in the last decades. It can be found mostly on the Holarctic region including 21 countries, which is supported by 51 publications. Its spreading mostly northward and eastward in the Palearctic is continuous due to anthropogenic effects, climate change as well as movement of infested materials. Flight phenology and population densities as a function of habitats were mapped. The detection in novel habitats of this pest due to imported wood materials and products is envisaged in the foreseeable future. The primary criterion for controlling the species adapted to the commercial trade and climatic change would be the elaboration of a monitoring system in affected and exposed areas.
\end{abstract}

Keywords Flight phenology $\cdot$ Global distribution $\cdot$ Meta-analysis $\cdot$ Neoclytus a. acuminatus $\cdot$ Population density $\cdot$ Range of expansion

\section{Introduction}

The red-headed ash borer Neoclytus acuminatus acuminatus (Fabricius 1775) (Coleoptera: Cerambycidae) is a woodboring pest posing major threat to the phytosanitary condition of wood products originating from forests and orchards (Csóka and Kovács 1999; Monné and Nearns 2020) in some regions. However, the beetle is not typically a pest throughout its entire range. So, in North America N. a acuminatus is not usually a pest in spite of its broad host range. Its phytosanitary status depends on the prevailing climate, the assortment of available host plants and the presence of its natural enemies (Estay et al. 2009). It is endemic to North America. (Linsley 1964). It was also accidentally introduced

Sándor Keszthelyi

ostrinia@gmail.com

1 Department of Agronomy, Hungarian University of Agriculture and Life Sciences, S. Guba str. 40, Kaposvár 7400, Hungary into the Adriatic region of southern Europe, where its range of occurrence continues to expand (Bense 1995).

The larvae develop in woody tissues of stressed and dead trees and can degrade hardwood lumber (Waters and Hyche 1984). The most common impact exerted by the pest on humankind is damage caused to felled trees intended for hardwood lumber or firewood. In natural circumstances, redheaded ash borer, as a saproxylophagous species, contributes to healthy forests by hastening the decomposition of dead and dying timber, making more nutrients and space available to healthy plants (Sama 2002; Brelih et al. 2006).

It is extremely polyphagous in deciduous trees (e.g. Acer, Betula, Castanea, Fagus, Juglans, Populus, Prunus, Quercus, Salix, Ulmus) and exceptional in conifers (Abies) (Brelih et al. 2006). The species will attack nearly all dying and dead hardwoods, but chiefly Fraxinus, Quercus, Carya, Diospyros, and Celtis. Unseasoned logs of Fraxinus, Quercus, and Carya with the bark intact are especially subject to heavy attack (Jurc et al. 2016).

It has a wide range of host plants, which makes it possible to find living conditions in diverse habitats. In addition, 
the invasiveness of the species was further strengthened by its vagility and good dispersion ability (Jurc et al. 2016), namely adults capable of flying great distances. The natural spread of $N$. a. acuminatus was unequivocally underscored by these latter facts. The species has excellently adapted to circumstances of freight by ship and plane, because the material of used empties is wood ensuring optimal conditions for the pest. The primary criterion for protection against the species would be the elaboration of a monitoring system in affected areas (Rassati et al. 2016).

The species overwinters as a larva, inside the sawn timber. Consequently, the life cycle of this beetle lasts for one year or (exceptionally) several years to complete. Adult $N$. a. acuminatus are present from March to late July (Bense 1995).

Neoclytus acuminatus acuminatus has well suited excellently to circumstances of freight by ship and plane, as the material of containers ensuring temperate microclimate used is generally wood, hence ensuring optimal conditions for the larvae. In addition, the active spreading via flying is a rather important factor for fast expansion (Rassati et al. 2016).

In spite of all these, special control procedures are not necessary since mainly dying or cut wood is attacked by $N$. a. acuminatus. Controls would be typical for other wood boring insects involving efforts to increase tree vigour through cultural practices, removal of infested wood prior to insect emergence, and treatment of trunks with protective insecticide sprays to coincide with egg laying (Brockerhoff et al. 2006).

The objective of this meta-analytic study was to map the worldwide distribution of N. a. acuminatus based on the processing of occurrence data of the species. Besides, among my goals was the determination of the theoretical directions of its spread in different continents as well as visualisation of the adults' seasonal activity and population density based on the online observation records. The acquired data can contribute to learn about the distribution ecology of invasive species through an understanding of the propagation characteristics of this insect.

\section{Materials and methods}

\section{Discovery of the current distribution}

All bibliographical references, phytosanitary reports and authentic photographic occurrences from the entomological websites in the quest of worldwide distribution of $N$. $a$. acuminatus have been tried to collect, which were available until August 2020. Several academic search engines [Google Scholar (GS), Web of Science (WoS), Scopus)] and international online insect- and photograph forums (alamy.com, anthrenus.pms-lj.si, BioLib.cz, BugGuide.net, bioras.petnica. rs entomologiitaliani.net, iNaturalist.org, inpn.mnhn.fr, izeltlabuak.hu, macroid.ru, BugGuide.net, whatsthatbug.com) provide primary help to the compilation of this extended database. To the scouting of the new habitats, I used besides the scientific name of the $N$. a. acuminatus, the terms "new/first", "data", "record", "observation" as well as "-interception" as keywords in the mentioned search engines. These observations were recorded and accurately placed on the map using Google Earth ${ }^{\circledR}$. The spatial distribution of the beetle was visualised by means of GIMP ${ }^{\circledR}$ (2018) (GNU Image Manipulation Program) and MapChart ${ }^{\circledR}$ (2020) software's.

Firstly, the native distribution area was reconfigured based on the studies of Hopping (1932) and Linsley (1964), in which the Nearctic region had formerly been indicated as a potential habitat of this species. Each data set was grouped by chronological order and used for assessing the occurrence of habitats and the present status of $N$. a. acuminatus. In addition, presumed directions of the spreading and actually occupied area of this arthropod were determined by means of further observational records of beetles. Thus, it became possible to prepare the global distribution chart of $N$. a. acuminatus over the whole Nearctic-Neotropical and western Palearctic ranges.

\section{Determination of seasonal activity and population density}

The seasonal activity of adult $N$. a. acuminatus' by states and countries by means of the data originating from the former listed online databases containing photographic (alamy.com etc.), observational (BugGuide.net, iNaturalist.org, etc.), and scholarly records (Scopus, WoS etc.) has been determined. The flight season of the year was divided according to the month's quarter marked by serial number (quarter I, II, III and IV). The main flight activity as the states and the countries of adults is shown by green bars. The calculations of the "main" flight activity period were determined by when $80 \%$ of all records of adult activity were found to occur. Furthermore, I have visualised the population density regarding the pest pressure based on the online observation records all over the world, which originated from the aforehand-mentioned entomological websites. The data were catalogued, and the population densities were mapped via red shades. For these calculations of population density $(d)\left(d=r / a \times 10^{4}\right)$, the number of observation records per unit area $(r)$ and the size of this area in $\mathrm{km}^{2}$ (a) were taken into consideration. 


\section{Results}

\section{Expansion and the directions of the global spreading of $\mathbf{N}$. a. acuminatus}

Table 1 contains the years of the first observations and present pest-status of $N$. a. acuminatus on the basis of published bibliographical references. Linked to this collection, online incidences are displayed in Table 2 and 3 (due to the large number of data in North America, only a summary table is included here), which completed this related database in whole Holarctic range. The expansion including the main parts of Nearctic and western Palearctic regions is conspicuous, but there are data from several Neotropical habitats as well in the last decades. Apart from Argentinean observations of this species, it can be found mostly on the northern hemisphere. Its presence has been shown in 21 countries (in 44 member states of the USA and 7 provinces of Canada), which is supported by 51 publications and several online reports of occurrences until now. It started to spread from Nearctic areas (where it was an endemic species), northwards, westwards and southwards, while it has reached several woody habitats in the Caribbean and Atlantic Maritime Ecozones. Besides, its western Palearctic spreading foci have encompassed Central Europe, the Balkans and Apennine peninsula from the Adriatic settlement point.

The distribution of the American continent as well as whole Holarctic expansion of $N$. a. acuminatus is shown in Fig. 1. The species is a typically Nearctic faunal element. The beetle is native in mid-east regions of the USA (more precisely New Jersey, Pennsylvania, Illinois, Kansas, Texas and Missouri states). In spite of this, the exact native area of this species cannot be determined, because more related observation records have already been available from different states for more than one century. Probably, the spreading in North-America supported by road traffic has intensified approximately since the beginning of twentieth century.

The beginning and exact time of the continuous south and westward expansion of the new North-American areas are rather obscure, but it is becoming mass and expansion of its Nearctic living place was unequivocally supported by intensifying urbanisation from the beginning of twentieth century. Accordingly, the species has got more observation data from the new habitats, for example from several points of Georgia and Florida states as well as from Idaho (in 1948). N. a. acuminatus became one of the most abundant longhorn beetle species in Alabama by 1979. The period of the next notable wave of the Nearctic expansion was in the years following right after the millennium when it has reached coasts of the Pacific Ocean and Atlantic Maritime Ecozones (2002-04). The beetle has presented itself almost all over the USA by now with the exception of Alaska, Montana, Wyoming, Nevada, and Arizona. Missing of the species in these states can be explained by unsuitable climate conditions. These newly occupied Nearctic areas can be assumed to be the primary source of further propagation in some Atlantic regions. Its global spreading started from these habitats, thanks to which $N$. a. acuminatus has been observed in Madeira Islands, the Antilles from the second quarter of twentieth century.

In South-America, N. a. acuminatus has been reported to be likely to be established in Argentina after two adults were collected in the field on planted trees before 1954 . This south Neotropical presence was confirmed by Di Iorio (2004) much later, who captured two males running on dead branches in the Central-Argentinean village, Bernasconi in 1998.

Accordingly, the first European observations include international harbours or aerodromes, where intense commercial activities were typical after the First World War. The oldest European findings originate from the Croatian city, Fiume (in 1851) (Fig. 2), starting from here with north-, west- and southward active spreading to Dalmatia, Istrian peninsula all the way to Montenegro as well as Po lowland and South Tirol in Italy. These regions can be considered as primary sources of spreading habitats, where its stable populations have been formed.

From this stabile propagation area, the beetle may have arrived through the Dinaric mountains and from the north, bypassing Slovenian and North-Croatian areas to Montenegro, Central-Serbia, and south-eastern Hungary. According to the work of Sama (2002), the most pronounced spreading directions of $N$. a. acuminatus in Europe nowadays are south- and eastward to the Balkan peninsula and Romania, and westward in the Carpathian-basin towards Transdanubia and South-Slovakia.

With regard to the westward direction, $N$. a. acuminatus has quickly occupied north Italy - where its southern border is Tuscany and Umbria in the Apennine peninsula—and reached the southern parts of Switzerland situated at the foot of Alps, Ticino and later Valais cantons. There are some records from the South of France, provinces of LanguedocRousillion, Alpes Azur and Rhone-Alps from the 1980s, but recent observations have not confirmed successful settlement in these areas since then.

In parallel to this, this species has emerged in other European locations at the beginning of the 20th century. One individual was reported by Buckle in 1902 in Belfast, which was undoubtedly imported in timber to islands of Ireland. Another, geographically separated focus is the Mark Brandenburg province in Germany, where a remarkable accumulation of $N$. a. acuminatus individuals has 
Table 1 The first observation data in order of appearance as well as the presence status on the World of Neoclytus acuminatus acuminatus based on bibliographical data

\begin{tabular}{|c|c|c|c|c|c|}
\hline & Country/State & Places of occurrence & Year & Presence status & References \\
\hline \multicolumn{6}{|c|}{ American continent and Atlantic regions } \\
\hline 1 & Portugal $(\mathrm{P})$ & Madeira Is.: Selvagens & 1937 & ep & $\begin{array}{l}\text { Picard (1937), Borges et al. (2008), } \\
\text { Monné and Nearns (2020) }\end{array}$ \\
\hline 2 & Florida (USA) & - & $<1940$ & & Hoffman (1940) \\
\hline 3 & Idaho (USA) & Boise, Nampa, Parma, Payette & 1948 & & Barr and Manis (1954) \\
\hline 4 & North Carolina (USA) & Cherokee county & 1965 & & Mount (1965) \\
\hline 5 & North Dakota (USA) & Minot & 1978 & & Kelly (1979) \\
\hline 6 & Cuba (C) & - & $<1983$ & oo & $\begin{array}{l}\text { Peck (2005), Nearns (2006), GBIF } \\
\text { (2019) }\end{array}$ \\
\hline 7 & Argentina (RA) & La Pampa (Bernasconi) & $<1954 ; 1998$ & & $\begin{array}{l}\text { Barr and Manis (1954), Di Iorio } \\
\text { (2004) }\end{array}$ \\
\hline 8 & Oregon (USA) & - & 2002 & ne & Nugent 2005 \\
\hline 9 & California (USA) & - & 2003 & & Seybold et al. (2016) \\
\hline 10 & Washington (USA) & - & 2003 & & USA Congress (2009) \\
\hline 11 & Canada (CDN) & $\begin{array}{l}\text { New Brunswick, Nova Scotia, Prince } \\
\text { Edvard Is }\end{array}$ & 2004 & oo & Webster et al. (2009) \\
\hline 12 & Puerto Rico (USPR) & Manati & 2007 & & Micheli (2007) \\
\hline 13 & Canada $(\mathrm{CDN})$ & $\begin{array}{l}\text { Southern Manitoba, Alberta, Sas- } \\
\text { katchewan }\end{array}$ & $?$ & & $\begin{array}{l}\text { Monné and Hovoré (2005), Monné } \\
\text { and Nearns (2020) }\end{array}$ \\
\hline 14 & Canada (CDN) & British Columbia & $?$ & & iNaturalist 2020 \\
\hline 15 & Mexico (MEX) & $\begin{array}{l}\text { Northern Coahuila, Nuevo Leon, } \\
\text { Tamaulipas }\end{array}$ & $?$ & & $\begin{array}{l}\text { Aguilar-Perez et al. (2007), Monné } \\
\text { and Nearns (2020) }\end{array}$ \\
\hline \multicolumn{6}{|c|}{ Europe } \\
\hline 16 & Croatia (HR) & Rijeka & 1851 & ep & Küster (1851) \\
\hline 17 & & Zadar & 1891 & & Jurc et al. (2016) \\
\hline 18 & North Ireland & Ulster (Belfast) & 1902 & oo & Buckle (1902) \\
\hline 19 & Italy (I) & Friuli-Venezia Giulia, Veneto & 1908 & ep & $\begin{array}{l}\text { Manzoni (1930), Winckler (1932), } \\
\text { Demelt (1950), (1956), Tassi } \\
\text { (1969), Sama (2002) }\end{array}$ \\
\hline 20 & Germany (D) & Mark Brandenburg & 1914 & & $\begin{array}{l}\text { Reineck (1919), Winckler (1932), } \\
\text { Hellrigl (1974) }\end{array}$ \\
\hline 21 & & Thuringia & 1929 & & Horion (1974) \\
\hline 22 & & Hesse, Rhineland-Palatinate & 1929-1951 & & Klausnitzer et al. (2016) \\
\hline 23 & Switzerland (CH) & Ticino & 1937 & & $\begin{array}{l}\text { Wittenberg (2005), Brelih et al. } \\
\text { (2006), Monnerat et al. (2015) }\end{array}$ \\
\hline 24 & Slovenia (SLO) & Gorizia, Coastal-Karst & 1949 & & Brelih et al. (2006) \\
\hline 25 & Czech Republic (CZ) & Karlovy Vary, Labem & 1951 & oo & Heyrovský (1951), Sláma (1998) \\
\hline 26 & Italy (I) & Trentino-Alto Adige & 1954 & ep & Horion (1975) \\
\hline 27 & Bosnia-Hercegovina (BIH) & Krajina, Tropolje, Hercegovina & 1973 & & $\begin{array}{l}\text { Mikšić and Georgijević (1973), } \\
\text { Mihajlović and Stanivuković (2009) }\end{array}$ \\
\hline 28 & France $(\mathrm{F})$ & Provence Alpes Azur, Rhone-Alps & 1976 & oo & $\begin{array}{l}\text { Sudre et al. (1999), Brustel et al. } \\
\text { (2002), Cocquempot and Lindelöw } \\
\text { (2010) }\end{array}$ \\
\hline 29 & & $\begin{array}{l}\text { Languedoc-Rousillion (Tharn } \\
\text { county) }\end{array}$ & 1980 & & Villiers (1979), Bijaoui (1980) \\
\hline 30 & Hungary $(\mathrm{H})$ & $\begin{array}{l}\text { Southern Great Plain, Central } \\
\text { Hungary }\end{array}$ & 1984 & ep & Csóka and Kovács (1999) \\
\hline 31 & Switzerland (CH) & Valais & 1992 & & $\begin{array}{l}\text { Wittenberg (2005), Brelih et al. } \\
\text { (2006), Cocquempot and Lindelöw } \\
\text { (2010), Monnerat et al. (2015) }\end{array}$ \\
\hline 32 & Hungary (H) & Transdanubia & 2002 & ne & Szeoke and Hegyi (2002) \\
\hline
\end{tabular}


Table 1 (continued)

\begin{tabular}{|c|c|c|c|c|c|}
\hline & Country/State & Places of occurrence & Year & Presence status & References \\
\hline 33 & United Kingdom (UK) & - & $<2002$ & oo & $\begin{array}{l}\text { Sama (2002), Monné and Nearns } \\
\text { (2020) }\end{array}$ \\
\hline 34 & Montenegro (MNE) & - & 2002 & ep & $\begin{array}{l}\text { Roganović (2007), Bulatović et al. } \\
\text { (2016) }\end{array}$ \\
\hline 35 & Serbia (SRB) & $\begin{array}{l}\text { Sumadija and Western Serbia (Mt. } \\
\text { Fruška Gora, Mt. Rtanj) }\end{array}$ & 2005 & & $\begin{array}{l}\text { Pil and Stojanovic (2005), Ilić and } \\
\text { Ćurčić (2013) }\end{array}$ \\
\hline 36 & Romania (RO) & $\begin{array}{l}\text { Western Romania: Timis county } \\
\text { (Timisoara) }\end{array}$ & 2005 & ne & Manci et al. (2005) \\
\hline 37 & Slovakia (SK) & Sturovo & 2008 & oo & Danilevsky (2014) \\
\hline 38 & Slovenia (SLO) & $\begin{array}{l}\text { Central Slovenia (Savinja: Brdo pri } \\
\text { Kranju) }\end{array}$ & 2012 & ne & Jurc et al. (2012) \\
\hline 39 & Serbia (SRB) & Central Serbia (Kragujevac) & 2014 & & Vukajlović and Živanović (2015) \\
\hline 40 & Austria (A) & Lower Austria (Theiss) & 2018 & oo, ne & Pennerstorfer and Kriechbaum (2018) \\
\hline
\end{tabular}

oo occasional occurrence, $e p$ established populations, ne newly emerged establishing populations

been registered since the beginning of the First World War. The beetle could spread westward to Thuringia, Hesse and Rhine-Palatine. According to Klausnitzer et al. (2016), occasional records were reported from these German provinces. The subsequent westward spread of the species has not reached France or the Benelux states, which is confirmed by the monograph survey of the Luxembourgian insect fauna of Vitali (2018).

Furthermore, a southward advance has been registered in the northern parts of the Czech Republic, where observational data originate from 1951. It should be mentioned, however, that other occurrence from this country has not been reported ever since up to now. The newest European observation data of species originate from north Austria from 2018, where some specimens were found in a fig tree. $N$. a. acuminatus is thought to have been introduced spontaneously through spreading from Germany or mediated by import trade activity from the American continent. Besides, it could inadvertently and occasionally have been imported, although apparently not established in the UK and France. Nevertheless, these records reveal the introduction of these beetles from North America to the European continent which still continues today.

\section{Seasonal activity and population density of N.a. acuminatus in the World}

It can be seen well the seasonal activity of the species depends on its distribution area characterising different climatic conditions (Fig. 3). However, the main period of adult appearance is between May and June independently of the observational location.

The southern regions both in Nearctic and in Palearctic areas can be characterised by longer adult flight seasons (period of adult activity), e.g. South West (from the middle of March until the end of August) and South East regions (from the end of March until the beginning of September) of the USA and the Mediterranean region of Europe (from the beginning of May until the end of July). In these areas, the appearances of $N$. a. acuminatus' adults are prolonged almost in the whole year, which is perfectly exemplified by both the Texan and the Oklahoman records, because in these warmer regions it may be present in the environment longer due to having multiple generations per year.

In contrast, the adult flight activities take place in narrower intervals - firstly at the end of spring and at the beginning of summer-in northern regions. This phenomenon was confirmed such as by Idahoan and Canadian records, where the flight is a rather shorter period, mainly from the middle of June to the end of July. Importantly, the higher population density areas of $N$. a. acuminatus in Nearctic coincide with its native distribution areas $(d=3.97-8.92)$ (Fig. 4). This phenomenon was especially proven by a high number of observational records in the east parts of the USA, e.g. Massachusetts $(d=20.31)$ or New Jersey $(d=18.34)$. A similar population density to these original distribution areas was observed in the main European propagation areas $(d=13,36)$ as well.

\section{Discussion}

Global expansion mostly in the northern hemisphere and other Atlantic areas of $N$. a. acuminatus was remarkable in the 20th century, which can be primarily explained by intensifying commercial and other transport activities (Pennerstorfer and Kriechbaum 2018). N. a. acuminatus has well suited to circumstances of freight by ship and plane, due to the fact that the main material of empties widely used are wood ensuring optimal conditions for the species (Rassati 
Table 2 European incidence of Neoclytus acuminatus acuminatus in online social networking sites and insect forums

\begin{tabular}{|c|c|c|c|c|}
\hline & Place of occurrence & Date & Photo by & URL \\
\hline \multicolumn{5}{|c|}{ Croatia } \\
\hline 1 & Istria & Unknown & Vaclav Hanzlik & https://www.biolib.cz/en/image/id39789/ \\
\hline 2 & Drenje & 29.06.2016 & Petr Horsák & https://www.biolib.cz/en/taxonimage/id296830/?taxonid=277626 \\
\hline 3 & Jadransko more & 14.08 .2019 & rubazin & https://www.inaturalist.org/observations/30798754 \\
\hline 4 & Zagreb & 26.06 .2020 & mpecarevic & https://www.inaturalist.org/observations/50992260 \\
\hline \multicolumn{5}{|c|}{ France } \\
\hline 5 & St-Armand Montrond & Unknown & tex-anne & https://inpn.mnhn.fr/espece/cd_nom/223101?lg=en \\
\hline \multicolumn{5}{|c|}{ Germany } \\
\hline 6 & Unknown & 13.03 .2015 & blickwinkel & $\begin{array}{l}\text { https://www.alamy.com/stock-photo-redheaded-ash-borer-red-headed-ash- } \\
\text { borer-red-headed-ash-borer-neoclytus- } 86064176 . h t m l\end{array}$ \\
\hline \multicolumn{5}{|c|}{ Hungary } \\
\hline 7 & Városerdő & 24.05 .2012 & Nagy Zoltán & https://www.izeltlabuak.hu/talalat/2653 \\
\hline 8 & Szakály & 25.05 .2014 & Balogh Diána & https://www.izeltlabuak.hu/talalat/21852 \\
\hline 9 & Martfú & 31.05 .2015 & Botka Tibor & https://www.izeltlabuak.hu/talalat/7012 \\
\hline 10 & Budapest & 07.05 .2015 & NagyS & https://www.izeltlabuak.hu/talalat/47817 \\
\hline 11 & Hortobágy & 30.06 .2015 & g_katona & https://www.inaturalist.org/observations/22748977 \\
\hline 12 & Budapest & 17.06.2016 & lmark & https://www.izeltlabuak.hu/talalat/4682 \\
\hline 13 & Bogyoszló & 19.06.2016 & adercsaba & https://www.izeltlabuak.hu/talalat/5631 \\
\hline 14 & Kalocsa & 22.05 .2017 & kanocpapa & https://www.izeltlabuak.hu/talalat/19260 \\
\hline 15 & Nádudvar & 27.05 .2017 & Sanyus & https://www.izeltlabuak.hu/talalat/7975 \\
\hline 16 & Tiszalök & 30.05 .2017 & futor & https://www.izeltlabuak.hu/talalat/8639 \\
\hline 17 & Szekszárd & 11.05 .2018 & novotnygreta & https://www.izeltlabuak.hu/talalat/42845 \\
\hline 18 & Öcsöd & 21.05 .2018 & u_1526153055 & https://www.izeltlabuak.hu/talalat/27483 \\
\hline 19 & Törökszentmiklós & 11.04 .2019 & KocsácsJudit & https://www.izeltlabuak.hu/talalat/63660 \\
\hline 20 & Dunatetétlen & 19.04.2019 & Marussi Istvánné & https://www.izeltlabuak.hu/talalat/86938 \\
\hline 21 & Szakály & 20.04.2019 & Balogh Diána & https://www.izeltlabuak.hu/talalat/61264 \\
\hline 22 & Baja & 21.04 .2019 & lofos & https://www.inaturalist.org/observations/22824636 \\
\hline 23 & Törökszentmiklós & 21.04 .2019 & Gabi80 & https://www.izeltlabuak.hu/talalat/70773 \\
\hline 24 & Kiskunhalas & 22.04 .2019 & drymar & https://www.izeltlabuak.hu/talalat/61887 \\
\hline 25 & Iváncsa & 26.04 .2019 & Benja_HUN & https://www.izeltlabuak.hu/talalat/62619 \\
\hline 26 & Téglás & 12.05 .2019 & Cilu005 & https://www.izeltlabuak.hu/talalat/66316 \\
\hline 27 & Domaszék & 18.05 .2019 & barnajanos & https://www.izeltlabuak.hu/talalat/93052 \\
\hline 28 & Ócsa & 18.05 .2019 & gammarusfarum & https://www.izeltlabuak.hu/talalat/72835 \\
\hline 29 & Abádszalók & 26.05 .2019 & Sylvia Deserti & https://www.izeltlabuak.hu/talalat/70991 \\
\hline 30 & Székesfehérvár & 03.06 .2019 & Hirvenkurpa & https://www.izeltlabuak.hu/talalat/71085 \\
\hline 31 & Kakasd & 07.06 .2019 & diocles66 & https://www.izeltlabuak.hu/talalat/72560 \\
\hline 32 & Kistarcsa & 11.06 .2019 & beta1 & https://www.inaturalist.org/observations/26803270 \\
\hline 33 & Mogyoród & 12.06 .2019 & TenPe & https://www.izeltlabuak.hu/talalat/73221 \\
\hline 34 & Harkakötöny & 16.06 .2019 & drymar & https://www.izeltlabuak.hu/talalat/74197 \\
\hline 35 & Törökbálint & 16.06 .2019 & Masska & https://www.izeltlabuak.hu/talalat/74200 \\
\hline 36 & Balatonalmádi & 22.06 .2019 & vargasz & https://www.izeltlabuak.hu/talalat/75926 \\
\hline 37 & Mogyorós & 22.06 .2019 & sirath & https://www.inaturalist.org/observations/27438349 \\
\hline 38 & Maglód & 02.07 .2019 & rekuci0120 & https://www.izeltlabuak.hu/talalat/78925 \\
\hline 39 & Budapest & 18.04 .2020 & exjunior & https://www.izeltlabuak.hu/talalat/108815 \\
\hline 40 & Gárdony & 01.05 .2020 & raerek & https://www.izeltlabuak.hu/talalat/109545 \\
\hline 41 & Csákvár & 02.05 .2020 & Sipi92 & https://www.izeltlabuak.hu/talalat/110200 \\
\hline 42 & Várdomb & 03.05 .2020 & Hirvenkurpa & https://www.izeltlabuak.hu/talalat/120720 \\
\hline 43 & Budapest & 07.05 .2020 & czimbalmostamás & https://www.izeltlabuak.hu/talalat/121448 \\
\hline 44 & Dunakeszi & 10.05 .2020 & rothbarna & https://www.izeltlabuak.hu/talalat/112183 \\
\hline 45 & Tiszalúc & 10.05 .2020 & babirusza & https://www.izeltlabuak.hu/talalat/112104 \\
\hline
\end{tabular}


Table 2 (continued)

\begin{tabular}{|c|c|c|c|c|}
\hline & Place of occurrence & Date & Photo by & URL \\
\hline 46 & Ócsa & 14.05 .2020 & Rahmé Nikola & https://www.izeltlabuak.hu/talalat/113454 \\
\hline 47 & Tiszasziget & 17.05.2020 & mataschek & https://www.izeltlabuak.hu/talalat/114317 \\
\hline 48 & Debrecen & 19.05.2020 & evikicsi2 & https://www.izeltlabuak.hu/talalat/114672 \\
\hline 49 & Budapest & 03.06 .2020 & Rapala Miklós & https://www.izeltlabuak.hu/talalat/120529 \\
\hline 50 & Magyaregregy & 05.06 .2020 & Bazsi18 & https://www.izeltlabuak.hu/talalat/155419 \\
\hline 51 & Budapest & 25.06.2020 & Xespok & https://www.izeltlabuak.hu/talalat/128745 \\
\hline 52 & Zalaszántó & 27.06.2020 & sampspade & https://www.izeltlabuak.hu/talalat/146754 \\
\hline 53 & Felsőtárkány & 28.06.2020 & ferati & https://www.izeltlabuak.hu/talalat/130372 \\
\hline 54 & Dombóvár & 02.07 .2020 & Huerequ & https://www.izeltlabuak.hu/talalat/130902 \\
\hline \multicolumn{5}{|c|}{ Italy } \\
\hline 55 & Pavia & 05.05 .2007 & Fumea Crassioralle & $\begin{array}{l}\text { http://www.entomologiitaliani.net/public/forum/phpBB3/viewtopic.php?f= } \\
\text { 145\&t=41655\&hilit=neoclytus }\end{array}$ \\
\hline 56 & Varenna & 20.06.2007 & roberto-brembilla & https://www.inaturalist.org/observations/10577800 \\
\hline 57 & Duino-Aurisina & 11.05.2008 & Bemisia & $\begin{array}{l}\text { http://www.entomologiitaliani.net/public/forum/phpBB3/viewtopic.php?f= } \\
145 \& \mathrm{t}=24928 \& \text { hilit=neoclytus }\end{array}$ \\
\hline 58 & Feltre & 12.06 .2010 & Maw86 & $\begin{array}{l}\text { http://www.entomologiitaliani.net/public/forum/phpBB3/viewtopic.php?f= } \\
\text { 145\&t=8678\&hilit=neoclytus }\end{array}$ \\
\hline 59 & Barolo & 25.05 .2012 & Davide & $\begin{array}{l}\text { http://www.entomologiitaliani.net/public/forum/phpBB3/viewtopic.php?f= } \\
\text { 145\&t=38722\&hilit=neoclytus }\end{array}$ \\
\hline 60 & Villa Estense & 13.06 .2013 & Marketto & $\begin{array}{l}\text { http://www.entomologiitaliani.net/public/forum/phpBB3/viewtopic.php?f= } \\
145 \& \mathrm{t}=45400 \& \text { hilit=neoclytus }\end{array}$ \\
\hline 61 & Strada Provinciale & 14.04.2017 & marcobertolini & https://www.inaturalist.org/observations/5685566 \\
\hline 62 & Casanova del Morbasco & 28.05.2017 & damighez & https://www.inaturalist.org/observations/6407414 \\
\hline 63 & Torino & 07.05 .2019 & vuillermoz & https://www.inaturalist.org/observations/24878283 \\
\hline 64 & Milano & 06.06 .2019 & lorenzo_bracchi & https://www.inaturalist.org/observations/26500250 \\
\hline 65 & Serravalle & 15.06.2019 & eleonora89 & https://www.inaturalist.org/observations/27016045 \\
\hline 66 & Province of Trento & 30.06 .2019 & apeterlongo & https://www.inaturalist.org/observations/28075337 \\
\hline 67 & Ferrara & 01.08 .2019 & valentinabuono & https://www.inaturalist.org/observations/29973257 \\
\hline 68 & Ferrara & 02.08.2019 & valentinabuono & https://www.inaturalist.org/observations/30164429 \\
\hline 69 & Boara Polesine & 03.09 .2019 & magister73 & https://www.inaturalist.org/observations/32053727 \\
\hline 70 & Rimini & 09.12 .2019 & farcippo & https://www.inaturalist.org/observations/36496267 \\
\hline 71 & Provinica de Ferrara & 19.05 .2020 & andreagrossi & https://www.inaturalist.org/observations/46652375 \\
\hline 72 & Bergamo & 20.05 .2020 & liampasto & https://www.inaturalist.org/observations/46637358 \\
\hline 73 & Como & 22.05 .2020 & paolamcasale & https://www.inaturalist.org/observations/46827901 \\
\hline 74 & Strada di Vigheffio & 04.06 .2020 & ciccoman & https://www.inaturalist.org/observations/48564981 \\
\hline 75 & Mornese & 10.06.2020 & germanoferrando & https://www.inaturalist.org/observations/52650826 \\
\hline 76 & Brescia & 19.06.2020 & jdtodi & https://www.inaturalist.org/observations/50140440 \\
\hline 77 & Monteroni d'Arbia & 26.06 .2020 & andrea_chemello & https://www.inaturalist.org/observations/50985514 \\
\hline 78 & Ferrara & 26.06.2020 & valentinabuono & https://www.inaturalist.org/observations/54419571 \\
\hline 79 & Ferrara & 08.08 .2020 & velentinabuono & https://www.inaturalist.org/observations/55842949 \\
\hline \multicolumn{5}{|c|}{ Montenegro } \\
\hline 80 & Kotor & 22.05 .2020 & viktor1608 & https://www.inaturalist.org/observations/48265775 \\
\hline \multicolumn{5}{|c|}{ Romania } \\
\hline 81 & Timiş & 06.05 .2019 & mishuherppunk & https://www.inaturalist.org/observations/24668955 \\
\hline 82 & Sfanta Elena & 20.06.2020 & standa & https://www.biolib.cz/en/taxonimage/id185390/?taxonid=277626 \\
\hline \multicolumn{5}{|c|}{ SERBIA } \\
\hline 83 & Unknown & 2009 & Dragiša Savić & http://bioras.petnica.rs/slika.php?id=518 \\
\hline 84 & Unknown & 2014 & Miloš Popović & http://bioras.petnica.rs/slika.php?id=10162 \\
\hline 85 & Unknown & 2014 & Ivan Pančić & http://bioras.petnica.rs/slika.php?id=9966 \\
\hline 86 & Novi Sad & 28.07.2014 & Geza Farkas & $\begin{array}{l}\text { http://www.photogeza.com/invertebrates/beetles/cerambycidae/slides/33_redhe } \\
\text { aded_ash_borer.html }\end{array}$ \\
\hline
\end{tabular}


Table 2 (continued)

\begin{tabular}{|c|c|c|c|c|}
\hline & Place of occurrence & Date & Photo by & URL \\
\hline 87 & Koviljski rit & 28.07.2014 & Geza Farkas & $\begin{array}{l}\text { http://www.photogeza.com/invertebrates/beetles/cerambycidae/slides/34_redhe } \\
\text { aded_ash_borer.html }\end{array}$ \\
\hline 88 & Unknown & 2016 & Zoran Gavrilović & http://bioras.petnica.rs/slika.php?id=15457 \\
\hline \multicolumn{5}{|c|}{ Slovenia } \\
\hline 89 & Brje pri Komnu & 03.05 .2002 & Matija Gogala & $\begin{array}{l}\text { http://anthrenus.pms-lj.si/animalia/galerija.php?id=PMSL-PZFN_Media- } \\
00364\end{array}$ \\
\hline 90 & Gračišče & 23.05.2013 & Miroslav Kastelic & $\begin{array}{l}\text { http://anthrenus.pms-lj.si/animalia/galerija.php?id=PMSL-PZFN_Media- } \\
00364\end{array}$ \\
\hline 91 & Tinjan & 02.06 .2018 & Mirsolav Kastelic & $\begin{array}{l}\text { http://anthrenus.pms-lj.si/animalia/galerija.php?id=PMSL-PZFN_Media- } \\
00364\end{array}$ \\
\hline 92 & Nova Gorica & 23.06.2020 & Janez Kamin & $\begin{array}{l}\text { http://anthrenus.pms-lj.si/animalia/galerija.php?id=PMSL-PZFN_Media- } \\
00364\end{array}$ \\
\hline
\end{tabular}

Table 3 Summarising North-American incidence of Neoclytus acuminatus acuminatus in online social networking sites and insect forums

\begin{tabular}{|c|c|c|c|c|c|c|c|c|c|}
\hline States of USA & iNaturalist.org & BugGuide.net & $\begin{array}{l}\text { what- } \\
\text { sthatbug. } \\
\text { com }\end{array}$ & $\operatorname{SUM}(\Sigma)$ & States of USA & iNaturalist.org & $\begin{array}{l}\text { Bug- } \\
\text { Guide. } \\
\text { net }\end{array}$ & $\begin{array}{l}\text { what- } \\
\text { sthatbug. } \\
\text { com }\end{array}$ & $\operatorname{SUM}(\Sigma)$ \\
\hline Alabama & 10 & - & - & 10 & New Jersey & 16 & 3 & 2 & 21 \\
\hline Arkansas & 5 & - & 1 & 6 & New Mexico & 17 & 1 & 1 & 19 \\
\hline California & 8 & - & 3 & 11 & New York & 25 & 2 & - & 27 \\
\hline Colorado & 9 & - & - & 9 & North Carolina & 23 & - & - & 23 \\
\hline Connecticut & 7 & 1 & - & 8 & North Dakota & 3 & - & - & 3 \\
\hline Delaware & 3 & - & - & 3 & Ohio & 40 & - & 1 & 41 \\
\hline Florida & 5 & - & 1 & 6 & Oklahoma & 16 & 1 & - & 17 \\
\hline Georgia & 8 & 2 & - & 10 & Oregon & 2 & - & - & 2 \\
\hline Idaho & 3 & - & - & 3 & Pennsylvania & 40 & 5 & 2 & 47 \\
\hline Illinois & 23 & 1 & - & 24 & Rhode Island & 3 & 1 & - & 4 \\
\hline Indiana & 7 & 2 & - & 9 & South Carolina & 10 & 3 & - & 13 \\
\hline Iowa & 5 & - & - & 5 & South Dakota & 2 & - & - & 2 \\
\hline Kansas & 16 & 2 & 1 & 19 & Tennessee & 10 & 1 & 1 & 12 \\
\hline Kentucky & 5 & 3 & - & 8 & Texas & 152 & 1 & 6 & 159 \\
\hline Louisiana & 18 & 2 & - & 20 & Utah & 12 & - & 2 & 14 \\
\hline Maine & 6 & 1 & - & 7 & Vermont & 5 & - & - & 5 \\
\hline Maryland & 11 & 4 & - & 15 & Virginia & 32 & 1 & - & 33 \\
\hline Massachusetts & 22 & 1 & - & 23 & Washington & 9 & 1 & 1 & 11 \\
\hline Michigan & 13 & - & - & 13 & West Virginia & 2 & 1 & - & 3 \\
\hline Minnesota & 7 & - & 1 & 8 & Wisconsin & 9 & 7 & 2 & 18 \\
\hline Mississippi & 7 & - & - & 7 & \multicolumn{5}{|c|}{ Provinces of Canada } \\
\hline Missouri & 6 & - & 1 & 7 & Manitoba & 3 & - & - & 3 \\
\hline Nebraska & 15 & - & - & 15 & New Brunswick & 8 & - & - & 8 \\
\hline New Hampshire & 5 & 1 & - & 6 & Quebec & 33 & - & - & 33 \\
\hline & & & & & Saskatchewan & 2 & - & - & 2 \\
\hline
\end{tabular}

et al. 2016). The rapid spreading was excellent supported by these special features. In addition, the continuous increase in flights as part of intensifying commercial activity is a rather important factor for the fast expansion provided that the prevailing temperature of its given distribution areas matches with that of its new habitats (Seybold et al. 2016). According to the study of Liebhold et al. (2013), the forest pest species are much more concentrated in the north-eastern region of the USA compared with other parts of the country. The similarity in historical spread among different types of 


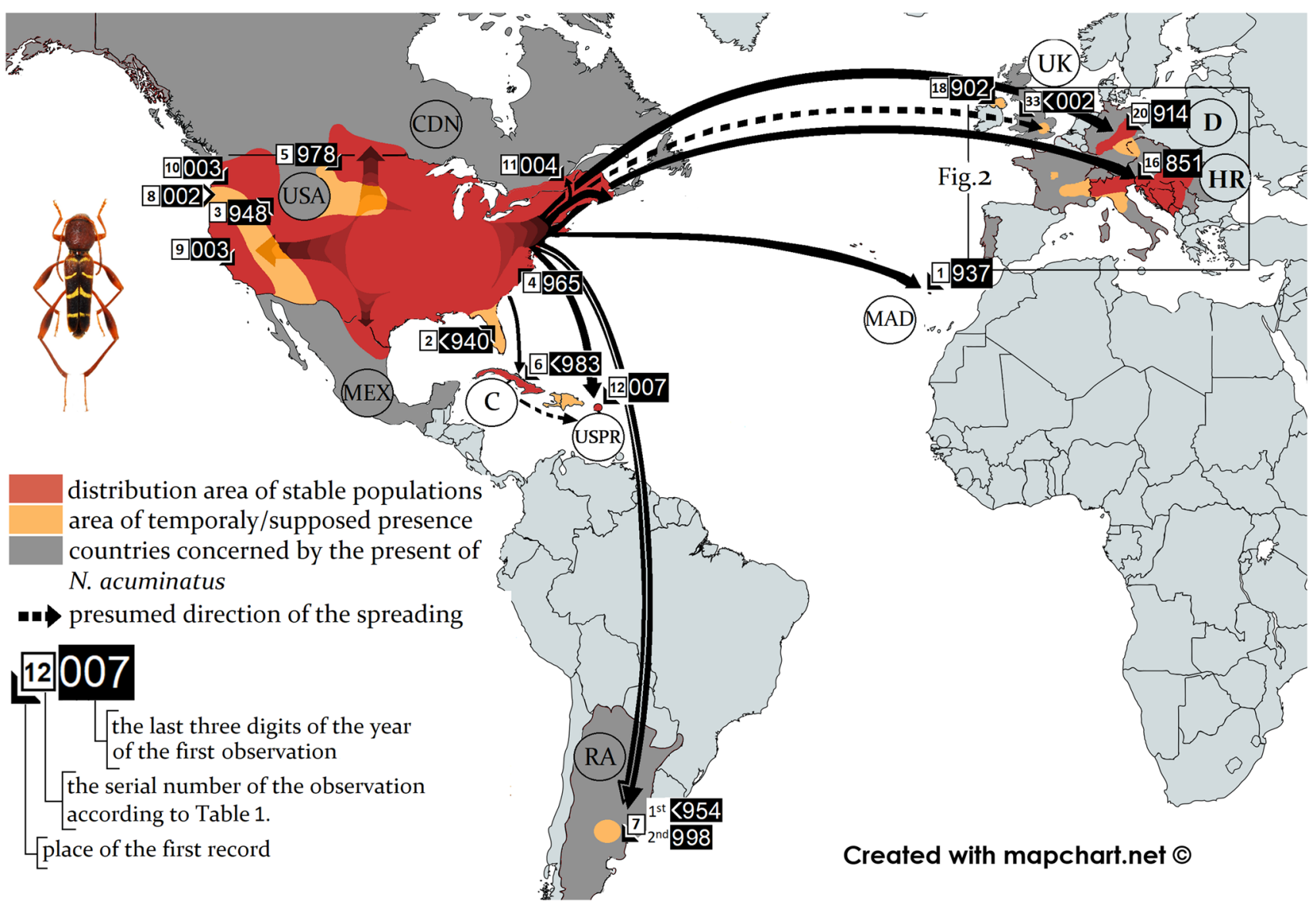

Fig. 1 Main first records, distribution and the theoretical spreading of Neoclytus acuminatus acuminatus on the world

organisms indicates the importance of anthropogenic movement in spread.

Hitherto, in the Nearctic areas N. a. acuminatus has already gained ground up to the northern boreal forests; only due amount of time is required for the formation of stable populations in these newly occupied areas. The fast process of this propagation will be supported by polyphagous features, ecological flexibility of the pest and the high diversity of woody habitats of these areas (Moné and Hovore 2005; Moné and Nearns 2020).

The Palaearctic and Neotropic introductions mediated by human activity of $N$. a. acuminatus are continuous until now, which is confirmed by more observational data from different parts of the world. Naturally, these introductions have not resulted in stabile populations in all cases, which are exemplified by Argentinean observation as well. It is not known whether some observed specimens originate from temporal introduction or a newly established population (Di Iorio 2004).

The "conquest" of the European continent by the species has been accomplished through air and water trades by import items originating from the USA. With the gradually intensified international transport and trading of commodities from 1850 to 1925 , well adaptive species-like $N$. $a$. acuminatus - have been able to reach Europe alive and become established (Reineck 1919; Sama 2002). To the emergence of the most extensive European propagation source originating from the Mediterranean basin, the optimal background was ensured by the optimal climate and the wide range of potential hosts. These regions have favoured the establishment of more alien species during the last century. For instance, the wood-boring beetles can be easily transported between continents by all kinds of woody materials (Rassati et al. 2016). The further European advance will be probably continued at fast pace in the future.

In general, it can be ascertained that the period of the main activity period of this species is from the beginning of May to the end of June. The prolonged adult activity of the southern regions leads to more generations developing in these areas, which is unequivocally explained by their higher effective temperature regimes (Linsley 1964; Sama 2002). The maximum of the seasonal activity of adult in some European habitats has not reached the native Nearctic observations yet, which is explained by both the differentiation of 


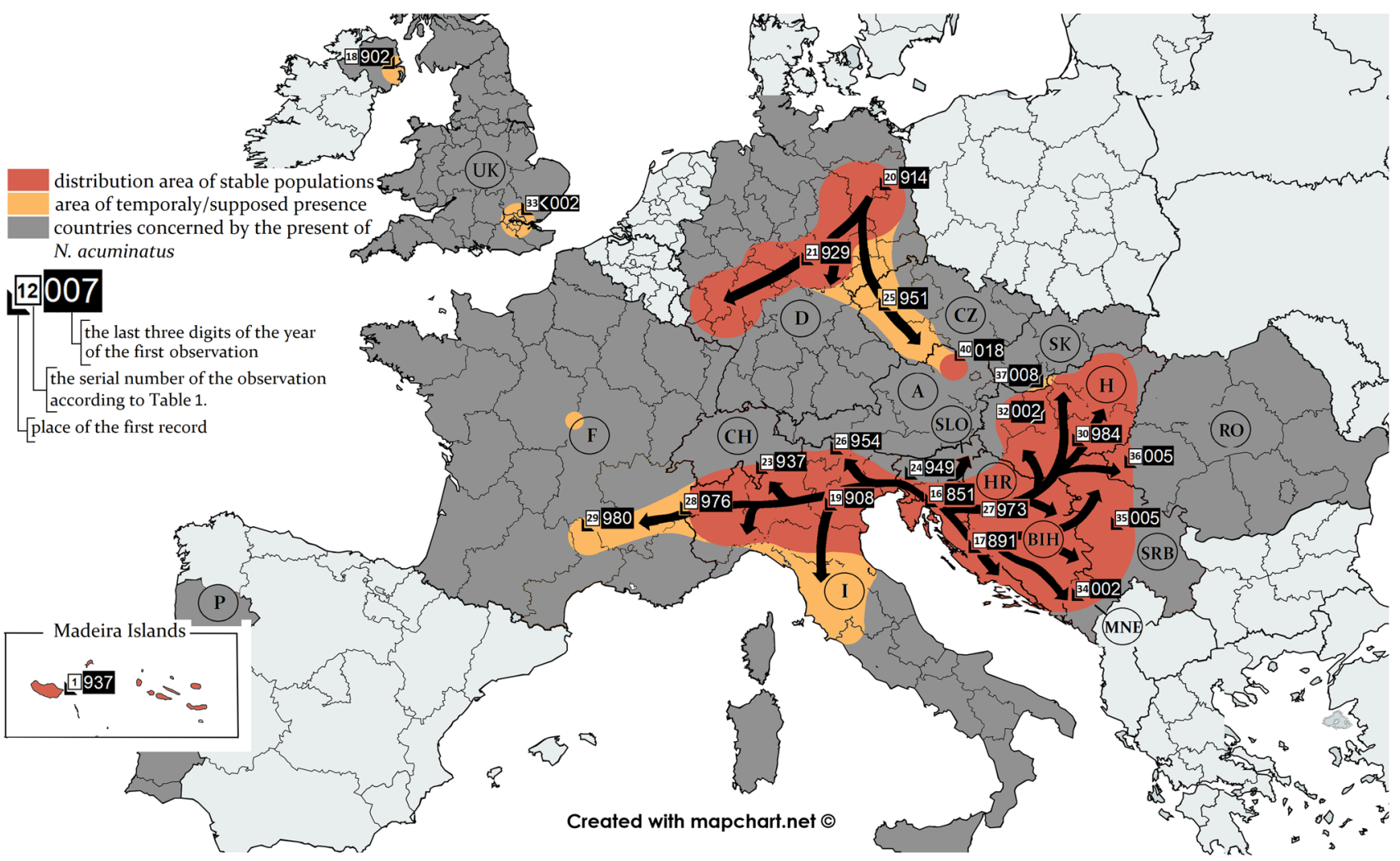

Fig. 2 Occurrence data, distribution and the theoretical spreading of Neoclytus acuminatus acuminatus in Europe

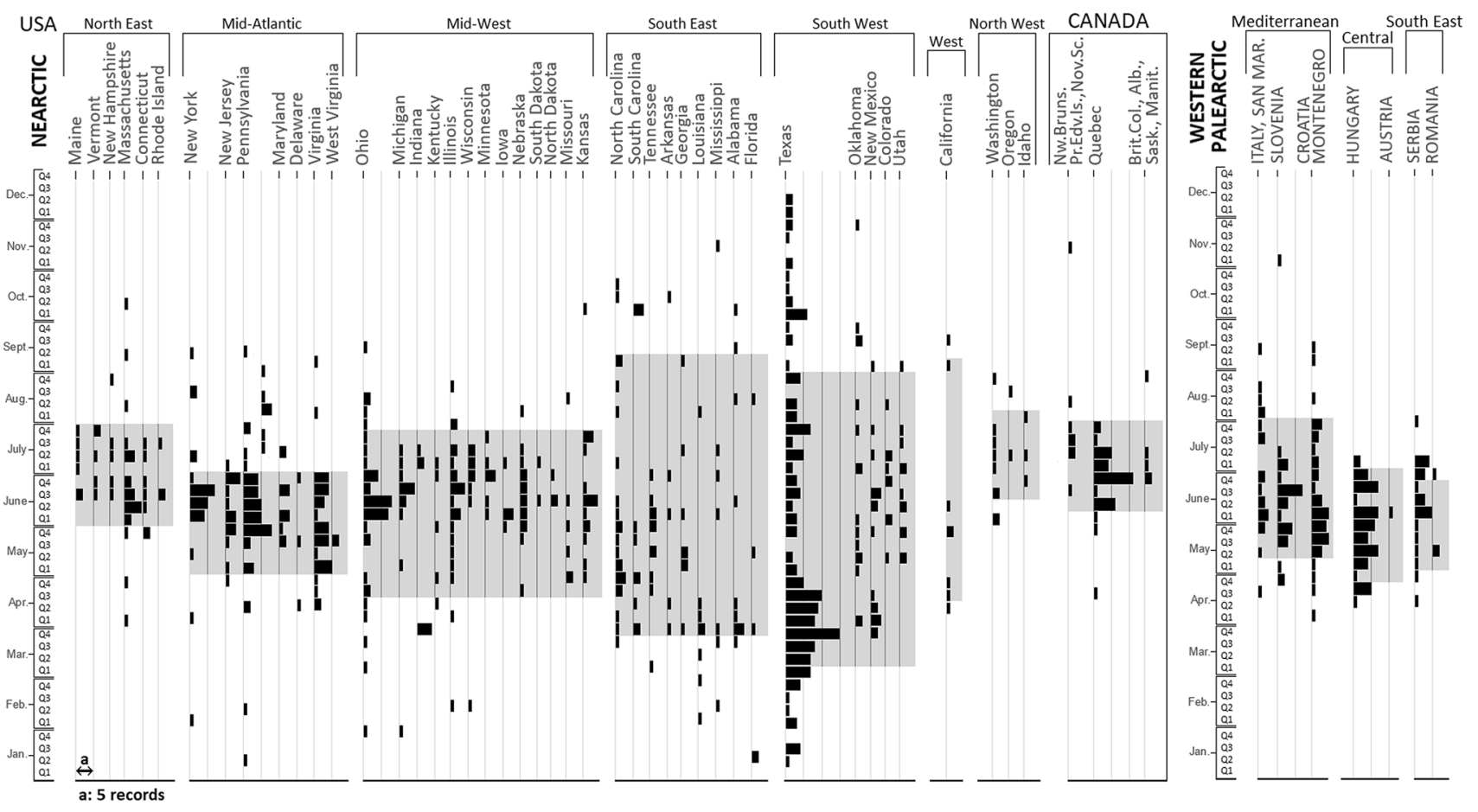

Fig. 3 Seasonal activity of Neoclytus acuminatus acuminatus as a function of the states and countries based on the online observation records 


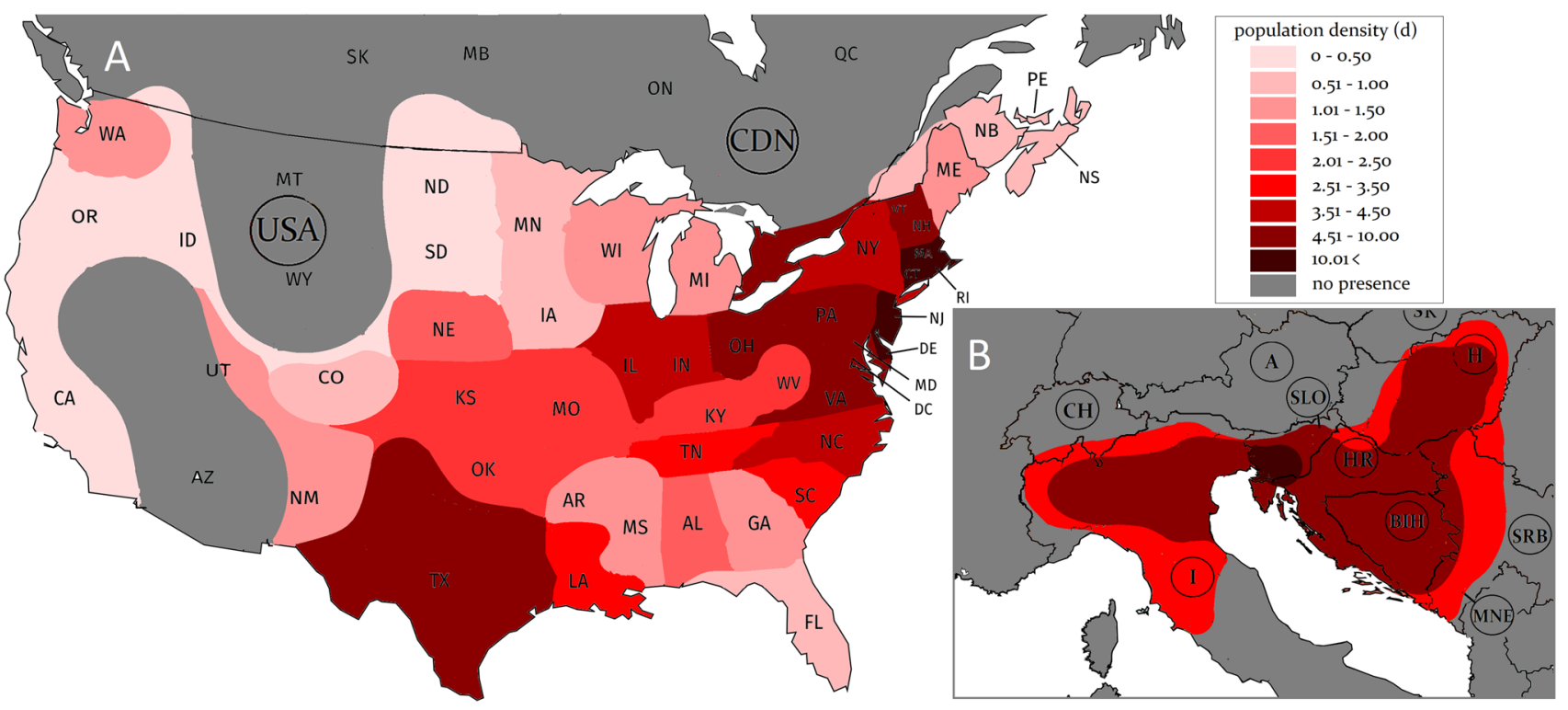

Fig. 4 The estimated population density in the Holarctic range based on the online observation records. a North-America; b: Europe

temperature amount and not stabilised populations in newly occupied areas.

The mid-west and the eastern regions of the USA are evaluated to be the first propagation areas of N. a. acuminatus. The calculated value in the newly occupied area is not represented by higher population density yet, which can be expounded by the ongoing advance of the species. The main European propagation areas can be characterised by a relatively high population density, which is explained by the fast-spreading of the species supported by beneficial climatic and ecological conditions (Westphal et al. 2008; Rassati et al. 2016).

Naturally, my applied method has primarily relied on the documented data sources, which can contribute to the approximate distribution of $N$. a. acuminatus. The main difficulty of such exploration is the searchability and the availability of local bibliographical data. The articles containing the first record of the newly emerged species were only published in a nationally refereed journal in several cases, which could even be written in Cyrillic and are not linked to international databases. The additional trouble with the online records, is that the species observed in the new habitats were poorly identified or uploaded because of the absence of a special entomological knowledge.

The presence of this species due to imported items is presumed in the foreseeable future. The primary criterion for controlling the species adapted to commercial trade and climatic change would be the elaboration of a monitoring system in affected and exposed areas. The aggregation pheromone of $N$. a. acuminatus has been already discovered (Lacey et al. 2004; Fan et al. 2019), which can help to the realisation of a basic global pest monitoring system.
The record registered by this international network is able to alarm about the presence of the new habitat and form a situation close to reality.

Acknowledgements I am indebted to the EFOP-3.6.3.VEKOP-16-2017-00008 and the EFOP-3.6.3-VEKOP-16-2017-00005 projects for the financial support. The project is co-financed by the European Union, the European Social Fund.

Funding Open access funding provided by Hungarian University of Agriculture and Life Sciences.

\section{Declarations}

Conflict of interest The author declare that I have no potential conflict of interest in relation to the study in this paper.

Open Access This article is licensed under a Creative Commons Attribution 4.0 International License, which permits use, sharing, adaptation, distribution and reproduction in any medium or format, as long as you give appropriate credit to the original author(s) and the source, provide a link to the Creative Commons licence, and indicate if changes were made. The images or other third party material in this article are included in the article's Creative Commons licence, unless indicated otherwise in a credit line to the material. If material is not included in the article's Creative Commons licence and your intended use is not permitted by statutory regulation or exceeds the permitted use, you will need to obtain permission directly from the copyright holder. To view a copy of this licence, visit http://creativecommons.org/licenses/by/4.0/.

\section{References}

Aguilar-Pérez H, Rodríguez-del-Bosque LA, Aguilar-Pérez CH, de la Peña ND (2007) Seasonal abundance of Xyleborus ferrugineus 
(Coleoptera: Curculionidae) on pecan trees in Northern Coahuila Mexico. Southwest Entomol 32(2):105-109

Barr WF, Manis HC (1954) The red-headed ash borer in Idaho. J Econ Entomol 47(6):1150

Bense U (1995) Longhorn beetles: illustrated key to the Cerambycidae and Vesperidae of Europe. Verlag Josef Margraf, Weikersheim

Bijaoui R (1980) Notes de chasses et observations diverses: Neoclytus acuminatus (Coleoptera: Curculionidae) dans le Tarn. L'Entomol $36: 268$

Borges PAV, Abreu C, Aguiar AMF, Carvalho P, Jardim R, Melo I, Oliveira P, Sérgio C, Serrano ARM, Vieira P (2008) A list of the terrestrial fungi, flora and fauna of Madeira and Selvagens archipelagos. Direcçao Regional do Ambiente de Madeira and Universidade dos Açores, Funchal and Angra do Heroísmo. Portugal.

Brelih S, Drovenik B, Pirnat A (2006) Material for the Beetle Fauna (Coleoptera) of Slovenia. 2nd contribution, Polyphaga: Chrysomeloidea (= Phytophaga): Cerambycidae). Scopolia 58:1-442

Brustel H, Berger P, Cocquempot C (2002) Catalogue des Vesperidae et des Cerambycidae de la faune de France (Coleoptera). Ann Soc Entomol Fr 38(4):443-461

Buckle CW (1902) Entomological notes from Ulster. Ir Nat 11(2):40-44

Bulatović I, Hadžiablahović S, Roganović D (2016) Montenegro national report of invasive alien species. http://esenias.org/files/ 06_Esenias_report_2016_73-78_Montenegro.pdf. Accessed 15 May 2020

Brockerhoff EG, Liebhold AM, Jactel H (2006) The ecology of forest insect invasions and advances in their management. Can J Forest Res 36:263-268

Cocquempot C, Lindelöw $\AA$ (2010) Longhorn beetles (Coleoptera, Cerambycidae). BioRisk 4:193-218

Csóka Gy Kovács T (1999) Xylophagous insects. Hungarian Forest Research Institute, Budapest

Danilevsky ML (2014) Longicorn beetles (Coleoptera, Cerambycoidea) of Russia and adjacent countries. Part 1. Moscow: HSC. (in Russian)

Demelt C (1950) Nota sul Neoclytus acuminatus Fabr. (Coleopt. Cerambycidae). Atti Soc Ital Sci Nat Mus Civ Stor Nat Milano 89:229-231

Demelt C (1956) Osservazioni sulla biologia e variabilitá del Neoclytus acuminatus F. (Col., Cerambycidae). Natura 47:36-39

Di Iorio OR (2004) Exotic species of Cerambycidae (Coleoptera) introduced in Argentina. Part 2. New records, host plants, emergence periods, and current status. Agrociencia 38(6):663-678

Estay SA, Lima M, Labra FA (2009) Predicting insect pest status under climate change scenarios: combining experimental data and population dynamics modelling. J Appl Entomol 133(7):491-499

Fan J, Denux O, Courtin C, Bernard A, Javal M, Millar JG, Hanks LM, Roques A (2019) Multi-component blends for trapping native and exotic longhorn beetles at potential points-of-entry and in forests. J Pest Sci 92:281-297

GBIF (2019) Neoclytus acuminatus (Fabricius, 1775) in GBIF Secretariat. GBIF Backbone Taxonomy. Checklist dataset. https:// doi.org/https://doi.org/10.15468/39omei. Accessed 27 July 2020

GIMP (2018) Gnu Image Manipulation Program. 2.10.8. Accessed $15 \mathrm{Jul} 2020$

Hellrigl K (1974) Nachtrag zur Cerambyciden-Fauna von Südtirol. Koleopterol Rundsch 51:32-55

Heyrovský L (1951) Catalogus Coleopterum Cechosloveniae. Supplementum. Cerambycidae (Tesaríci). Acta Soc Entomol Cech 48:43-53

Hoffman CH (1940) Additions to annotated lists of insects reared from elm bark and wood. Bull Brooklyn Entomol Soc 35:54-63
Hopping G (1932) A revision of the Clytini of boreal America (Cerambycidae, Coleoptera). Part 1. Ann Entomol Soc Am 25:529-567

Horion A (1974) Faunistik der mitteleuropäischen Käfer, Bd. XII: Cerambycidae, Bockkäfer (mit 52 Verbreitungskarten). ÜberlingenBodensee, Germany

Horion A (1975) Nachtrag zur Faunistik der mitteleuropäischen Cerambycidae. Nachr B1 Bayer Ent 24(6):97-115

Ilić N, Ćurčić S (2013) The longhorn beetles (Coleoptera: Cerambycidae) of Rtanj Mountain (Serbia). Acta Entomol Serb 18(1/2):69-94

Jurc M, Bojovik S, Fernández MF, Jurc D (2012) The attraction of cerambycids and other xylophagous beetles, potential vectors of Bursaphelenchus xylophilus, to semiochemicals in Slovenia. Phytoparasitica 40:337-349

Jurc M, Csóka G, Hrašovec B (2016) Potentially important insect pests of Celtis australis in Slovenia. Croat Hung Šum List 140(11-12):577-588

Kelly G (1979) Redheaded ash borer in North Dacota. Anim Plant Health Insp Serv. US Dept Agric Coop Plant Pest Rep 4(1):1-25

Klausnitzer B, Klausnitzer U, Wachmann E, Hromadko Z (2016) Die Bockkäfer Mitteleuropas, vol 2. Die Neue Brehm Bücherei, Germany

Küster HC (1851) Die Käfer Europa's. Nach der Natur beschrieben. Mit Beiträgen mehrerer Entomologen, vol 22. Bauer \& Raspe, Germany

Liebhold AM, McCullough DG, Blackburn LM, Frankel SJ, Von Holle B, Aukema JE (2013) A highly aggregated geographical distribution of forest pest invasions in the USA. Biodiv Res 19(9):1208-1216

Linsley EG (1964) The Cerambycidae of North America, Part V. Univ Calif Publ Entomol 20:1-197

Lacey ES, Ginzel MD, Millar JG, Hanks LM (2004) Male-produced aggregation pheromone of the cerambycid beetle Neoclytus acuminatus acuminatus. J Chem Ecol 30:1493-1507

Manzoni L (1930) A new pest of the vine, Neoclytits acuminatus. F Gior Agric Dom 40(45):582

MapChart (2020) Software for the graphical presentation of linkage maps and QTLs. 2.10.8. https://mapchart.net/. Accessed 10-25 July 2020

Micheli JA (2007) Neoclytus acuminatus (Fabricius), (Coleoptera: Cerambycidae) in Puerto Rico: new Caribbean record. Coleopt Bull 61(4):502

Mihajlović LJ, Stanivuković Z (2009) Allochthonous insect species on forest and ornamental woody plants in the Republic Srpska. Glas Šumar Fak Univ Banjoj Luci 11:1-26

Mikšić RI, Georgijević E (1973) Cerambycidae Jugoslavije II deo. Akademija nauka i umjetnosti Bosne i Hercegovine, Djela, knj.XLVII. Odelj Pri Mat Kih Nauka 4:1-153

Manci OD (2005) Neoclytus acuminatus (Fabricius, 1775) new genus and species for Romanian fauna (Coleoptera: Cerambycidae: Cerambycinae). Bul Inf Entomol 16:75-77

Monné MA, Hovore FT (2005) Checklist of the Cerambycidae (Coleoptera) of the Western Hemisphere. BioQuip Publications, Rancho Dominguez

Monné MA, Nearns EH (2020) Catalogue of the Cerambycidae (Col.) of Canada and United States of America. https://cerambycids. com/catalog/Monne\&Nearns_2020_NearcticCat_part_III.pdf. Accessed 28 July 2020

Monnerat C, Chittaro Y, Sanchez A, Gonseth Y (2015) Liste commenteé des Lucanidae, Cetoniidae, Buprestidae et Cerambycidae (Coleoptera) de Suisse. Bull Soc Entomol Suis 88:173-228

Mount DA (1965) Red-headed ash borer in South Carolina. Animal and plant health inspection service. US Dept Agric Coop Plant Pest Rep 15(1):282 
Nearns EH (2006) A checklist of the Cerambycidae (Coleoptera) holdings of the Fernando de Zayas collection, Havana Cuba. Coleopt Bull 60(1):53-57

Peck SB (2005) A checklist of the beetles of Cuba with data on distributions and bionomics (Insecta: Coleoptera). Arthr Fla Neighb Areas 18:1-241

Pennerstorfer J, Kriechbaum M (2018) First record of Neoclytus acuminatus (Fabricius, 1775) (Coleoptera: Cerambycidae) for Austria. Beitr Entomofaun 19:133-145

Picard F (1937) Sur les Cérambycides de Madere et des îles voisines. C R Seances Soc Biol 13:70-72

Pil N, Stojanovic D (2005) New longhorn beetles (Coleoptera: Cerambycidae) from Serbia and Montenegro. Arch Biol Sci 57(2):143-147

Reineck G (1919) Die Insekten der Mark Brandenburg II. Coleoptera, Cerambycidae, Dtsch Entomol Z 62:1-92

Rassati D, Lieutier F, Faccoli M (2016) Alien wood-boring beetles in Mediterranean regions. In: Paine T, Lieutier F (eds) Insects and diseases of mediterranean forest systems. Springer, Cham, pp 293-327

Roganović D (2007) Contribution to the knowledge of alien insect species in Montenegro. In: Tomov R, Trenchev M, Trencheva K (eds) Proceedings of the international conference: alien arthropods in South East Europe. crossroad of three continents. 19-21 Sept 2007 Sofia, Bulgaria, p 56

Sama G (2002) Atlas of the Cerambycidae of Europe and Mediterranean area. Vol. 1: Northern, Western, Central and Eastern Europe. Zlín, Kabourek, Czech Republic

Seybold SJ, Penrose RL, Graves AD (2016) Invasive Bark and Ambrosia Beetles in California Mediterranean forest ecosystems. In: Paine T, Lieutier F (eds) Insects and diseases of mediterranean forest systems. Springer, Cham, pp 583-662

Sláma M (1998) Tesaříkovití-Cerambycidae České republiky a Slovenské republiky (Brouci-Coleoptera). Czech Republic, Krhanice

Sudre J, Foucart A, Cocquempot C (1999) Catalogue commenté et étude bibliographique des Coléoptères Cerambycidae du département de l'Hérault. B Mens Soc Linn Lyon 68(6):133-192

Szeőke K, Hegyi T (2002) A new grapevine pest: redheaded ash borer (Neoclytus acuminatus Olivier). Növényvédelem 38:268-269
Tassi F (1969) Un longicorne australiano per la prima volta in Italia (Col. Cerambycidae). Boll Assoc Rom Entomol 24:69-71

USA Congress (2009) Agriculture, rural development, food and drug administration, and realated agencies appropriations for 2009 . https://www.congress.gov/bill/111th-congress/house-bill/2997. Accessed 25 July 2020

Villiers A (1979) Un nouveau Clytini en France? L'Entomol 35:144

Vitali F (2018) Atlas of the insects of the Grand-Duchy of Luxembourg: Coleoptera, Cerambycidae Ferrantia 79. Musée national d'histoire naturelle, Luxembourg

Vukajlović F, Živanović N (2015) The longhorn beetles (Coleoptera: Cerambycidae) of the city of Kragujevac: Central Serbia. Kragujev J Sci 37:149-160

Waters DJ, Hyche LL (1984) Notes on Cerambycidae (Coleoptera) collected on dead or stressed deciduous trees in east central Alabama. Coleopt Bull 38(3):283-286

Webster RP, McCorquodale DB, Majka CG (2009) New records of Cerambycidae (Coleoptera) for New Brunswick, Nova Scotia, and Prince Edward Island, Canada. Biodiversity, Biosystematics and Ecology of Canadian Coleoptera II (ed. by C.G. Majka and J. Klimaszewski). ZooKeys 22:285-308

Westphal MI, Browne M, MacKinnon KN, Noble I (2008) The link between international trade and the global distribution of invasive alien species. Biol Invasions 10:391-398

Winkler A (1932) Catalogus Coleopterum regionis palaearcticae. Albert Winkler Verlag, Wien

Wittenberg R (2005) An inventory of alien species and their threat to biodiversity and economy in Switzerland. CABI bioscience Switzerland centre, Report to Swiss Agency for environment, forest and landscape. Délmont, Switzerland

Publisher's Note Springer Nature remains neutral with regard to jurisdictional claims in published maps and institutional affiliations. 